\title{
Improved Preoperative Planning in Robotic Heart Surgery
}

\author{
R Bauernschmitt ${ }^{1}$, M Feuerstein ${ }^{1}$, EU Schirmbeck ${ }^{1}$, J Traub ${ }^{2}$, \\ G Klinker ${ }^{2}$, SM Wildhirt ${ }^{1}$, R Lange ${ }^{1}$ \\ ${ }^{1}$ German Heart Center Munich, Munich, Germany \\ ${ }^{2}$ Institute for Informatics I, Technical University, Munich, Germany
}

\begin{abstract}
Optimal port placement and intraoperative navigation in robotically assisted heart surgery improve the efficiency of a telemanipulator system. A new interface for preoperative interactive port placement on a 3D-plot of the patient's thorax was established. A multislice CT scan of the thorax with applied landmarks is performed. The CT segmentation and reconstruction is achieved by the visualization tool amira ${ }^{T M}$ extended by a new module enabling the preoperative planning.Offline the instruments and the camera are placed virtually visualizing the optimal position. Simultaneously the system passes through the collision detection mode.

An accurate spatial registration of the anatomy is realized. The telemanipulated endoscopic camera and instruments are placed precisely as intended. Collision detection methods ensure an intersection-free planning and proper placement of the telemanipulator arms to access accurately the desired region of interest.
\end{abstract}

\section{Introduction}

In teleoperator based minimally invasive cardiovascular surgery, only small incisions (also referred to as ports) are used to perform an operation. Open heart surgery can be avoided, resulting in less surgical complications than in conventional invasive surgery [1].

However, current teleoperator systems still have some limitations [2]. First of all, it is difficult for the surgeon to place the teleoperator arms in a way that the whole operation region can be reached during the intervention. Frequently it is necessary to replace the ports during the operation to continue. Due to the limited field of view of the endoscopic camera, the surgeon lacks a larger overview over the operating region inside the patient. This makes navigation difficult, especially when the teleoperator tips are outside the view of the endoscope. We propose an approach for optimal port placement and intra-operative navigation in robotically assisted cardiac surgery in order to improve the efficiency of a teleoperator system. Our system was designed in close interdisciplinary collaboration of surgeons and computer scientists to aid the entire clinical workflow for robotically assisted cardiac surgery from the planning phase up to the actual intervention.

\section{Methods}

For the virtual simulation of an intervention, the teleoperator arms as well as assistance arms can be interactively placed and moved within the patient's model in such a way that they neither intersect with each other nor with vitals and bones. The planning tool's graphical user interface (Fig. 1) provides various visualization, planning, and validation options. Computed tomography

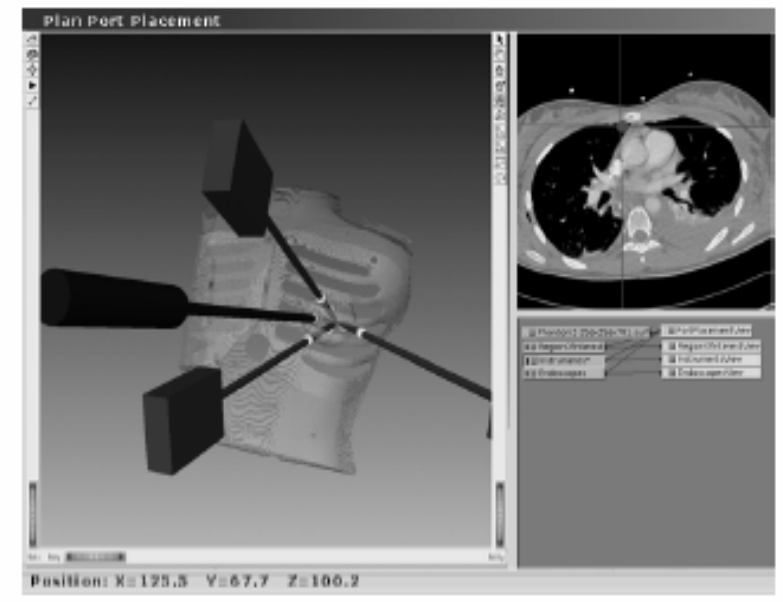

Fig. 1: User interface of the planning system

slices can be displayed in sagittal, coronal, and frontal directions and are automatically aligned with the reconstructed model in a common coordinate system. For a more intuitive planning procedure, the patient's model as well as the teleoperator arms can be made semitransparent or entirely hidden to see the patient's interior (Fig. 2). 


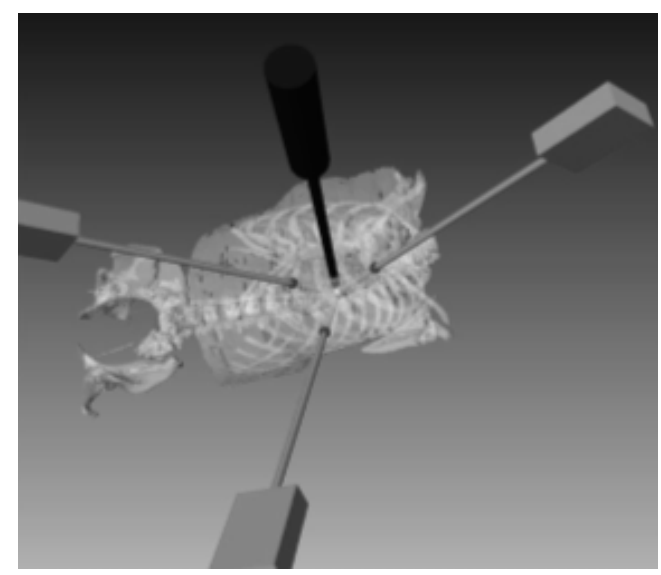

Fig. 2: Semitransparent patient model

Collision detection techniques and a virtual endoscope view support the verification of all poses of the teleoperator arms and their corresponding ports. Whereas collisions between teleoperator arms are avoided by realtime computations, collisions between arms and anatomy are detected by a fast triangle-triangle intersection detection algorithm applied on the patient's polygonal model. Certain anatomical structures such as the left lung, which is usually deflated during the intervention, or the patient's skin can be hidden and excluded from these collision detection checks. Similar to its application in neurosurgery, a virtual endoscope simulates exactly the view as obtained by the endoscope arm's camera by setting the estimated parameters. It can be used to simulate critical steps of the intervention in advance, to test the reachability of the whole working field. The planned position of the camera and the instruments are exported to the intra-operative port placement and navigation tool.

Augmented reality techniquesenable the projection of the previously planned optimal poses for the port placement on top of the real world view of the patient. The physicians are provided with an expedient visualization interface to see inside the patient. The image data is registered in three dimensions with the patient's thorax in order to perfectly align the modalities reality and virtuality. Fiducials (i.e. artificial landmarks) are placed onto the patient's skin before the computed tomography scan is performed. By using ink tattoos, the exact location of these fiducials can be reconstructed in the surgery room (Fig. 3).

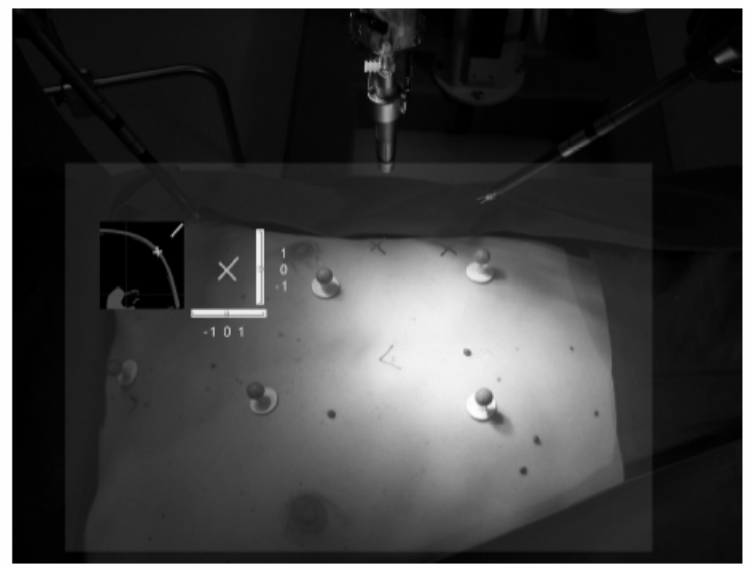

Fig. 3: A projector is mounted on the ceiling of the operating theater and the placement of the ports is guided by projecting the required information directly onto the patient's skin.

An infrared tracking system ${ }^{1}$ detects the position of the fiducials in the operating room at exactly the same location as detected in the computed tomography scan. A point based algorithm uses the corresponding position of the fiducials in both modalities reality and virtuality to compute the affiliated rigid transformation from one modality into another. Matching the three-dimensional model data with the patient during the surgery is done fully automatic based on a RANSAC approach. It selects a random minimal subset of correspondences to compute the rigid transformation. The pre-operatively planned port position is then superimposed on top of a video image of the real patient after a rigid spatial registration.

The endoscopic camera and instrument arms are tracked using the same infrared tracking system the patient is registered in. The tracking does not rely on the actual teleoperator parameters as in related approaches and therefore additional assistance arms that are placed independently of the teleoperator can be tracked. Furthermore, the proposed system can be used for verifying the mechanical tracking data provided via the teleoperator API (application programming interface).

The position data can be used as a supplementary source of feedback during the placement process in order to compensate the missing third dimension on the image plane by color augmentation. In addition, the pose of the tracked instruments can be used for intra-operative navigation, where the position of the tracked arms is augmented on the three-dimensional reconstructed model of the patient's thorax in real time. This can be applied in a totally virtual environment or by using augmented reality techniques.

${ }^{1}$ smARTtrack1 by A.R.T GmbH, www.ar-tracking.de 
Throughout the entire intervention the instrument arms can be tracked using the infrared tracking system. Thus, the position and orientation of the instrument arms can be visualized in real time in the reconstructed threedimensional model of the computed tomography scan of the patient's thorax.

In a visionary scenario, the limited field of view provided by the endoscopic camera may also be augmented. While other approaches try to integrate the augmentation into the video frame, for instance to identify coronary vessels, we propose to extend the surgeon's view such that around the video image the reconstructed structures from the pre-operative data are displayed together with the tracked teleoperator arms. This may improve the surgeons' orientation and allow the surgeon to see the position of the instrument tips even when they are not visible inside the actual endoscopic image, which can be used to guide the instruments to the region of interest.

\section{Results}

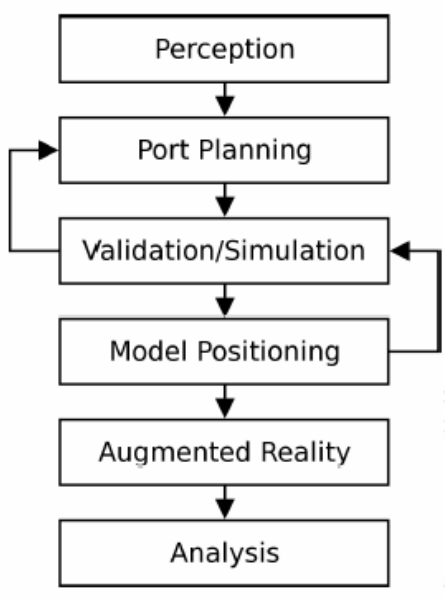

Fig. 4: Iterative steps of constructing an augmented reality-based system for port placement and intraoperative navigation based on the „amira“ - framework. Perception: creation of a virtual three-dimensional model of the patient and the telemanipulator arms and its visualization on any display. Port planning: the process of finding adequate incision sites for the telemanipulator arms Validation and simulation: techniques to validate the planned incision sites or the quality of positioning the model, respectively. Model positioning: the virtual model containing all planned data is positioned in the real world's operating theater. Augmented reality techniques: combining the virtual model with tracked data of the real world. Analysis: improving the process by identifying, rating, and evaluating
The operational steps are depicted in figure 4. After marking the region of interest, the surgeon is able to determine the optimal ports for the endoscopic camera and up to three instrument arms (Fig. 5a).

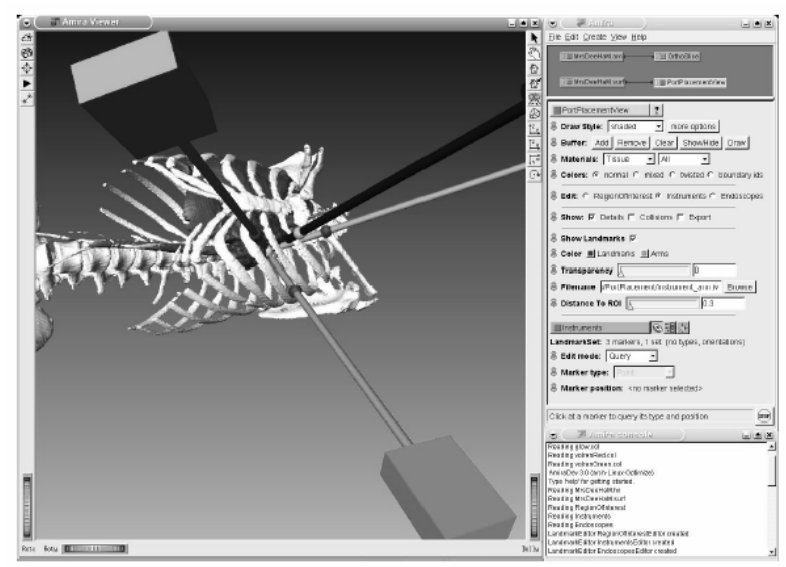

Fig. 5a: After 3-D-reconstruction of the individual patient's CT-data, the surgeon defines a region of interest. The model proposes one port for the endoscopic camera and up to three ports for the instrument arms based on empirical data, accessibility to the region of interest and the avoidance of collisions with vital organs, bones or among the instruments themselves. Within the model, all arms can be virtually moved as desired.

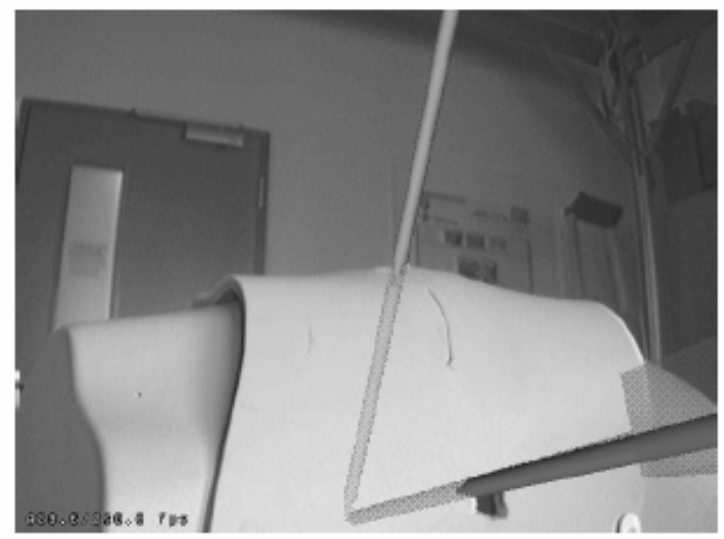

Fig. 5b: After tracking the chest model by infrared markers and an external camera system, ports and instruments are projected upon the surface of the chest model to determine the calculated incisions for port placement. 
These virtual arms can be integrated into the real world by superimposing them on the view of an external camera (Fig. 5b). The main planning functionality is achieved by a module called PortPlacementView, which is derived from the original amira ${ }^{\mathrm{TM}} \mathrm{HxDisplaySurface}$ module and thus inherits all of its features. Moreover, many additional amira $^{\mathrm{TM}}$ ports have been implemented to enable the users to perform the planning process in an intuitive way. The user is able to switch to a endoscopic view, meaning that the virtual camera is positioned exactly on top of the endoscope arm and aligned with its direction.

In order to track the telemanipulator arms in real time, infrared markers are attached to the instruments and the „patient“. The navigation application tracks the position of the instrument arms in the real world (Fig. 6) and projects their current position into the three-dimensional reconstructed model based on computed tomography images in real time. This offers the surgeon the possibility to estimate position and movements of the entire instrument without using the endoscopic camera.

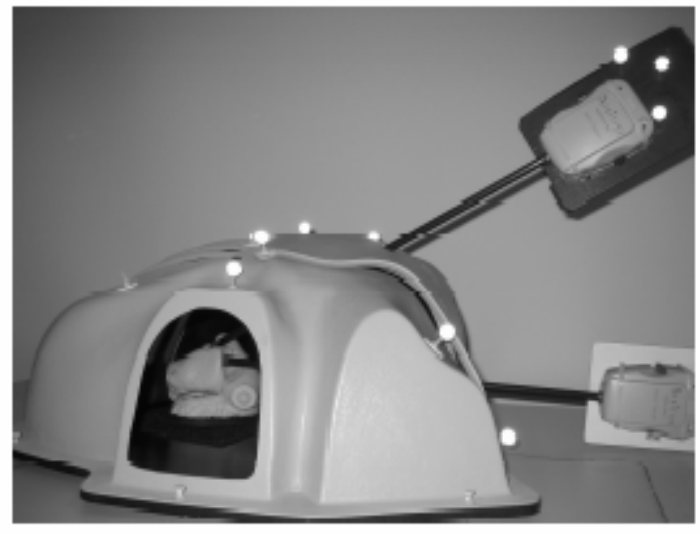

Fig. 6: „Patient“ (chest phantom) and instruments are eqipped with infrared markers to allow tracking by an external camera system.

\section{Discussion and conclusions}

Within the scope of this work a fully functional prototype for port placement and navigation in robotically assisted minimally invasive cardiovascular surgery was developed based on amira ${ }^{\mathrm{TM}}$ and DWARF. An accurate rigid spatial registration of the pre-operative acquired imaging data of the patient's anatomy is realized. Thus, the endoscopic camera and instruments are placed as intended. Currently, there are several similar systems for pre-operative planning and navigation in endoscopic surgery.
The research group ChIR has a focus on planning and simulation of robotic procedures, where models of deformable organs are created and augmented reality techniques are used to enable real-time and safe integration [3].

As an official consultant of Intuitive Surgical ${ }^{\circledR}$, the ChIR team is able to access the internal parameters of the da Vinci $^{\circledR}$ telemanipulator via its application programmer's interface. Thus, the exact positions and orientations of the telemanipulator arms and their tool tips can be computed using forward computation and be used for the planning process. However, compared to our system, ChIR is unable to plan any robotic intervention except those that are da Vinci ${ }^{\circledR}$ based, while our system is not dependent on a special robot or telemanipulator.

BrainLAB proposes an application in combination with an optimal cancer treatment planning tool to achieve high accuracy in tumour irradiation [4]. There is no described scenario in the cardiovascular or any other strongly deformable surgical field.

The systems proposed by Integrated Surgical Systems (ORTHODOC $^{\circledR}$ ) and Praxim-Medivision [5] are mainly elaborated for surgery of non deformable structures.

The future vision of augmentation in telesurgery is mapping of the real endoscopic view into the corresponding image plane of the endoscope in the virtual model, providing improved overview and navigation. Surgeons should be able to navigate the instrument arms into the desired field, even if not visible by the endoscopic camera.

\section{References}

[1] Boyd WD, Stahl KD. The Janus syndrome: a perspective on a new era of computer-enhanced robotic cardiac surgery. J Thorac Cardiovasc Surg. 2003 Sep;126(3):62530.

[2] Mohr F, Falk V, Diegeler A et al: Computer-enhanced robotic cardiac surgery: experience in 148 patients. J Thorac Cardiovasc Surg 2001; 121: 842-53

[3] Coste-Maniere E, Adhami L, Mourgues F et al: Optimal planning of robotically assisted heart surgery. Lecture notes in control and information sciences, 2002

[4] Soete G, v.d.Steene J, Verellen D et al: Clinical use of stereoscopic X-ray positioning of patients treated with conformal radiotherapy for prostate cancer. Int J Radiation 2002, 54 (3): 948-52

[5] Paleogolos S, Wadley JP, Kitchen ND et al: Clinical utility and cost-effectiveness of interactive image-guided meningeoma surgery. Neurosurgery 2000; 47: 40

Address for correspondence

Robert Bauernschmitt, MD, Ph.D.

German Heart Center, Lazarettstr. 36, 80636 Munich, Germany E-mail: Bauernschmitt@dhm.mhn.de 